\title{
Overexpression of Toll-Like Receptor 4 Affects Autophagy, Oxidative Stress, and Inflammatory Responses in Monocytes of Transgenic Sheep
}

\author{
Sutian Wang ${ }^{1,2 t}$, Xuting Song ${ }^{1 t}$, Kunli Zhang ${ }^{3}$, Shoulong Deng ${ }^{4}$, Peixin Jiao ${ }^{1}$, Meiyu Qi ${ }^{5}$, \\ Zhengxing Lian ${ }^{6}$ and Yuchang Yao ${ }^{1 *}$ \\ ${ }^{1}$ College of Animal Science and Technology, Northeast Agricultural University, Harbin, China, ${ }^{2}$ State Key Laboratory of \\ Livestock and Poultry Breeding, Guangdong Key Laboratory of Animal Breeding and Nutrition, Institute of Animal Science, \\ Guangdong Academy of Agricultural Sciences, Guangzhou, China, ${ }^{3}$ Institute of Animal Health, Guangdong Academy of \\ Agricultural Sciences, Key Laboratory of Livestock Disease Prevention of Guangdong Province, Scientific Observation and \\ Experiment Station of Veterinary Drugs and Diagnostic Techniques of Guangdong Province, Ministry of Agriculture and Rural \\ Affairs, Guangzhou, China, ${ }^{4}$ Chinese Academy of Sciences (CAS) Key Laboratory of Genome Sciences and Information, \\ Beijing Institute of Genomics, Chinese Academy of Sciences, Beijing, China, ${ }^{5}$ Institute of Animal Husbandry, Heilongjiang \\ Academy of Agricultural Sciences, Harbin, China, ${ }^{6}$ Beijing Key Laboratory for Animal Genetic Improvement, National \\ Engineering Laboratory for Animal Breeding, Key Laboratory of Animal Genetics and Breeding of the Ministry of Agriculture, \\ College of Animal Science and Technology, China Agricultural University, Beijing, China
}

Edited by:

Jiyan Zhang,

Independent Researcher, Beijing,

China

Reviewed by:

Vladimir Trajkovic,

University of Belgrade, Serbia Shengtao Zhou,

West China Second University Hospital, Sichuan University, China

*Correspondence: Yuchang Yao

yaoyc@neau.edu.cn

tThese authors have contributed equally to this work

Specialty section:

This article was submitted to Cell Death and Survival,

a section of the journa Frontiers in Cell and Developmental Biology

Received: 13 December 2019 Accepted: 24 March 2020 Published: 05 May 2020

Citation: Wang S, Song X, Zhang $K$,

Deng S, Jiao P, Qi M, Lian Z and Yao Y (2020) Overexpression of Toll-Like Receptor 4 Affects Autophagy, Oxidative Stress, and Inflammatory Responses in Monocytes of Transgenic Sheep.

Front. Cell Dev. Biol. 8:248.

doi: 10.3389/fcell.2020.00248
Toll-like receptor 4 (TLR4) is a critical pattern recognition receptor that plays a critical role in the host innate immune system's recognition of Gram-negative bacteria. Since it is the lipopolysaccharide (LPS) receptor, it links the activated inflammatory response with autophagy and oxidative stress. Autophagy, or type II programmed cell death, was reported to have defensive functions in response to the production of inflammatory cytokines and oxidative stress. To explore the relationship between autophagy, inflammation, and oxidative stress, a TLR4-enriched transgenic (Tg) animal model (sheep) was generated. Autophagy activity in the Tg blood monocytes was significantly higher than in the wild-type animal under LPS stress, and it returned to normal after transfection of TLR4 siRNA. Pretreatment with 3-methyladenine (3-MA) inhibited autophagy and enhanced oxidative stress and the production of TNF- $\alpha$. The LPSinduced reactive oxygen species (ROS) level was markedly increased in the Tg group at an early stage before quickly returning to normal values. In addition, suppressing ROS production by $\mathrm{N}$-acetyl-L-cysteine down-regulated the number of intracellular autophagosomes and the expression of Beclin-1, ATG5, and cytokines IL-1 $\beta$, IL-6, and TNF- $\alpha$. Further mechanistic investigation suggested that the TLR4-associated p38 mitogen-activated protein kinase (MAPK) signaling pathway was involved in autophagy and oxidative stress. P38 MAPK promotes intracellular autophagy, ROS production, and inflammatory response. Moreover, TLR4 over-expression suppressed oxidative stress and the production of inflammatory cytokines and increased autophagy activity in vivo. Taken together, our results showed that LPS induced autophagy, which was related to TLR4-mediated ROS production through the p38 MAPK signaling pathway. In addition, our study also provided a novel transgenic animal model to analyze the effects of TLR4 on autophagy, oxidative stress, and inflammatory responses.

Keywords: TLR4, autophagy, oxidative stress, inflammatory responses, transgenic animal model, p38 MAPK 


\section{INTRODUCTION}

Pattern recognition receptors (PRRs) play important roles in the recognition of microbial molecules by the animal's innate immunity (Janeway and Medzhitov, 2002). These molecules, termed pathogen-associated molecular patterns (PAMPs), can activate multiple inflammatory signaling pathways to clear the infection (Takeuchi and Akira, 2010). Toll-like receptors (TLRs) are PRRs found mainly on membranes (Chen et al., 2009). An essential element of this family is Toll-like receptor 4 (TLR4), which mainly recognizes lipopolysaccharide (LPS), one of the inflammatory PAMPs that exist in the cell walls of most Gramnegative bacteria. After the host has been infected with these bacteria, multiple oxidation intermediates and inflammatory cytokines can be activated through TLR4 (Ryan et al., 2004). TLR4 interacts with other proteins to form a TLR4/MD2/CD14 complex that can bind to LPS (Hoareau et al., 2010). Then, TLR4 recruits adaptors to trigger myeloid differentiation primary response gene 88 (MyD88)-dependent and MyD88-independent signaling (Tse et al., 2014), ultimately leading to the production of reactive oxygen species (ROS) and inflammatory responses.

Generally, ROS is maintained at a certain level to induce an oxidative reaction that helps to clear pathogenic bacteria, but an excess of oxidative stress will cause damage to the host. It has been shown that LPS-generated ROS and nicotinamide adenine dinucleotide phosphate oxidase (NOX) are closely related to ROS (Park et al., 2006; Yu et al., 2015). NOX1, NOX3, NOX4, NOX 5, DUOX1, and DUOX2 are collectively known as the NOX family proteins, which mainly exist in phagocytes and can produce ROS through the transfer of electrons. TLR4 downstream signaling, namely, the interleukin-1 receptor-associated kinase4 (IRAK-4), is involved in controlling NOX (Pacquelet et al., 2007). However, some research suggested that the TLR4-NOX2/4 signaling pathway influences the activated state of autophagy and inflammatory factors (Suzuki et al., 2012; Sciarretta et al., 2014). Thus, ROS may be involved in the communication between TLR4 and autophagy.

Essentially, autophagy is a lysosomal degradation process that can eliminate damaged organelles and pathogenic bacteria to maintain cell homeostasis and survival (Singh and Cuervo, 2011). It has been reported that the antioxidant reaction and autophagy that were induced under oxidative stress helped to concomitantly down-regulate ROS levels, inflammatory responses, and oxidative damage to organelles (Kim et al., 2017). It must be emphasized that this repair system enables the cell to reach a new homeostasis. Although these studies suggested a close relationship between ROS and autophagy, the interconnectedness between TLR4, ROS, the inflammatory response, and autophagy remains to be addressed in detail, which was the goal of this study.

TLR4 participates in the innate immunity response by mainly sensing LPS and then activating a variety of antimicrobial immune responses. Our previous studies have shown that, compared to the control cells, the peripheral blood monocytes from Tg sheep overexpressing TLR4 evoked a stronger inflammatory response under LPS stress at an early stage and dropped quickly back to initial levels (Deng et al., 2012).
In addition, the increased NO secretion induced a stronger intracellular oxidative stress that was due to the overexpression of TLR4 (Deng et al., 2015). Furthermore, the overexpression of TLR4 contributed to the phagocytosis of pathogenic bacteria via the p38 mitogen-activated protein kinase (MAPK) and phosphatidylinositide3-kinase (PI3K) signaling pathways (Wang et al., 2018a,b). Here we continued this line of investigation by first measuring the effects of TLR4 (overexpression and inhibition) on the interactions between oxidative stress and autophagy. Then, the inflammatory responses during TLR4mediated oxidative reaction and autophagy were assessed. Finally, the antioxidant NAC and autophagy inhibitor 3methyladenine (3-MA) were used to analyze the deep molecular mechanisms under the TLR4-mediated LPS stress. We present the first investigation of the interconnectedness between TLR4, ROS, inflammatory response, and autophagy in a Tg model overexpressing TLR4.

\section{MATERIALS AND METHODS}

\section{Animal Ethics Statement}

All the animal experiments and treatments followed the guidelines of the Animal Welfare Committee of the Northeast Agricultural University, and all the experiments were approved by the Animal Welfare Committee of the Northeast Agricultural University.

\section{Production and Detection of Tg Sheep}

$\mathrm{Tg}$ sheep were produced by transferring the linearized vector (digested with AseI) into the pronuclei of fertilized eggs by microinjection (Figure 1A). Generally, the transformed exogenous TLR4 genes were detected by Southern blot. The genomic DNA was extracted from peripheral blood monocytes. HindIII (NEB) was used to digest genomic DNA. The probe was generated by PCR with the following primer pair: forward (F), 5'-ACTGGTAAAGAACTTGGAGGAGG-3' and reverse (R), 5'-CCTTCACAGCATTCAACAGACC-3', producing a 671-bp amplicon that was labeled by digoxigenin (Roche). The total RNA of peripheral blood monocytes was extracted with TRIzol reagent (Invitrogen), and the PrimeScript RT Reagent Kit (TAKARA) was used to prepare the cDNA. The expression of TLR4 mRNA was measured by quantitative real-time PCR (qRT-PCR) via the ABI 7500 system with SYBR Premix Ex Taq II kit (TAKARA) according to the instructions. $\beta$-Actin was chosen to normalize the data of each sample. The TLR4 and $\beta$-actin primer sequences were as follows: TLR4, (F) 5'-ATCATCAGCGTGTCGGTTGTCA-3' and (R) $5^{\prime}$-GCAGCCAGCAAGAAGCATCAG-3 ${ }^{\prime} ; \beta$-actin, (F) $5^{\prime}$-AGATGTGGATCAGCAAGCAG-3' and (R) $5^{\prime}$ CCAATCTCATCTCGTTTTCTG-3'. The relative expression of mRNA was calculated by the $2^{-\Delta \Delta C T}$ method.

\section{Sheep Peripheral Blood Monocyte Isolation and Culture}

Sheep were divided into two groups: Tg sheep and wild-type (WT) sheep ( $n=3$ in each group). Sheep peripheral blood 


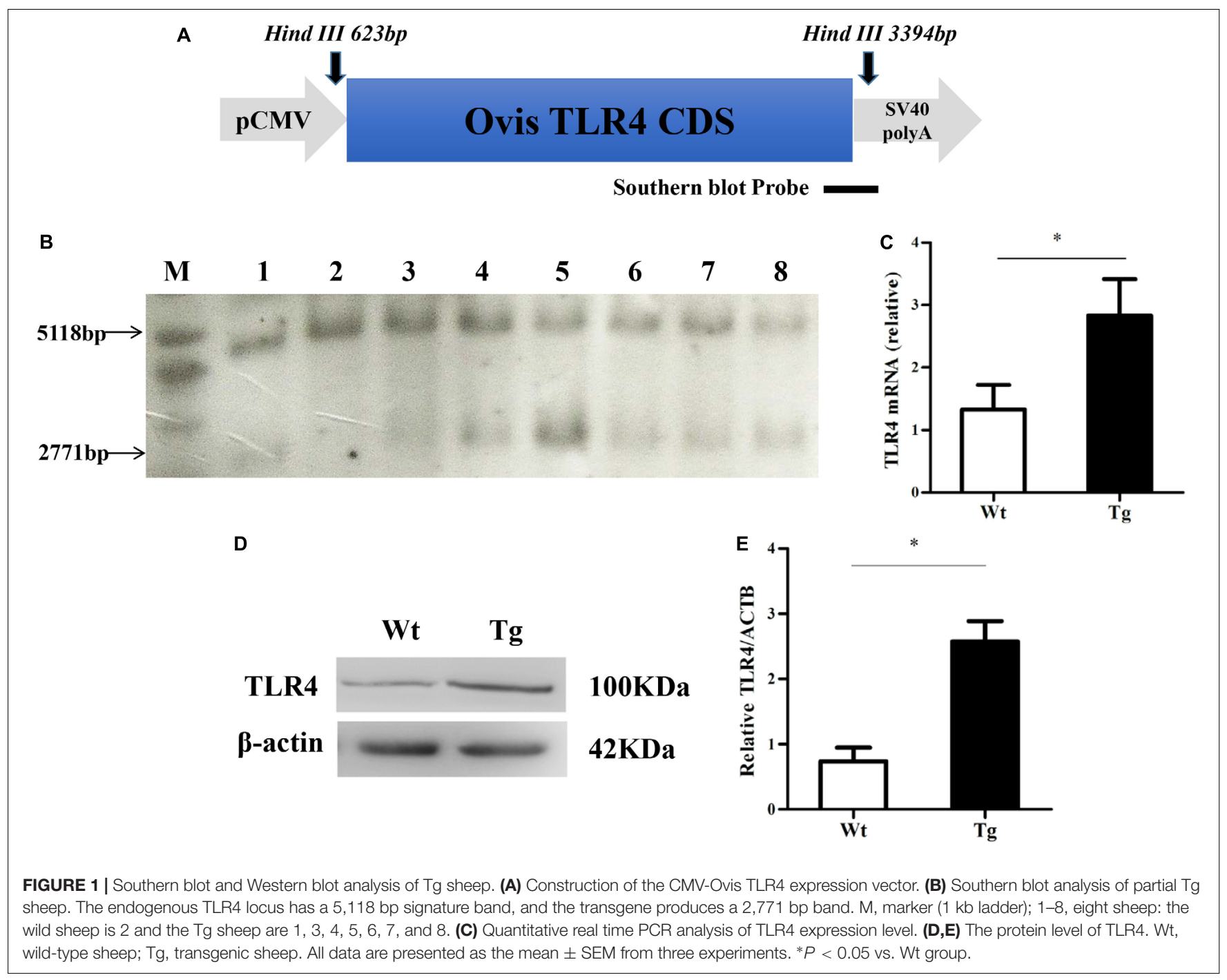

monocytes were isolated from the blood of sheep using the separation medium (Tbdscience). The cells were incubated at $37^{\circ} \mathrm{C}$ in a $5 \%-\mathrm{CO}_{2}$ incubator for $2 \mathrm{~h}$ and then the non-adherent cells were washed out. The adherent cells were cultivated in RPMI1640 (Gibco) containing 10\% fetal bovine serum (Gibco) at $37^{\circ} \mathrm{C}$ in a $5 \%-\mathrm{CO}_{2}$ incubator.

\section{Western Blotting}

The cells were harvested and lysed using RIPA buffer (Beyotime) with protease inhibitor cocktail and PMSF (Roche). Then, the proteins were quantified using the BCA Protein Assay Kit (Thermo Fisher Scientific). Equal amounts of proteins were resolved on $12 \%$ SDS-PAGE and transferred to a polyvinylidene fluoride membrane (Millipore). After incubation with primary antibodies against TLR4 (1:1,000; Affinity, AF7017), LC3B (1:1,000; Abcam, ab51520), ATG5 (1:1,000; Sigma, A0856), Beclin-1 (1:1,000; CST, 3495), actin (1:5,000; CST, 4970), GAPDH (1:5,000; Proteintech, 10494-1-AP), and horseradish peroxidaseconjugated secondary antibodies (1:1,000; Beyotime, A0208), the membranes were visualized by enhanced chemiluminescence
(Thermo Fisher Scientific). The protein bands were analyzed by ImageJ software (National Institutes of Health; version 1.45).

\section{Transfection of Small Interfering RNA}

To knock-down the expression of TLR4, sheep peripheral blood monocytes were transfected with siRNA-specific TLR4 from Genepharma (si-TLR4-86: sense, 5'-GCGU ACAGGUUGUUCCUAATT- $3^{\prime}$ and antisense, 5'-UUAGGAAC AACCUGUACGCTT-3'). Transfection was accomplished with lipofectamine RNAiMAX (Invitrogen) according to the manufacturer's instructions.

\section{Transmission Electron Microscopy}

The monocytes were treated with LPS (100 ng/ml) (Sigma, L6529) for $12 \mathrm{~h}$, and then the cells were collected to measure the autophagy level by transmission electron microscopy. In inhibitory experiments, the cells were pretreated with $10 \mathrm{mM}$ 3-MA for $6 \mathrm{~h}$, and LPS (100 $\mathrm{ng} / \mathrm{ml})$ was added for another $12 \mathrm{~h}$. Briefly, the monocytes were collected and fixed in 2.5 glutaraldehyde for $24 \mathrm{~h}$ and then in $1 \%$ osmic acid for $1 \mathrm{~h}$. The 


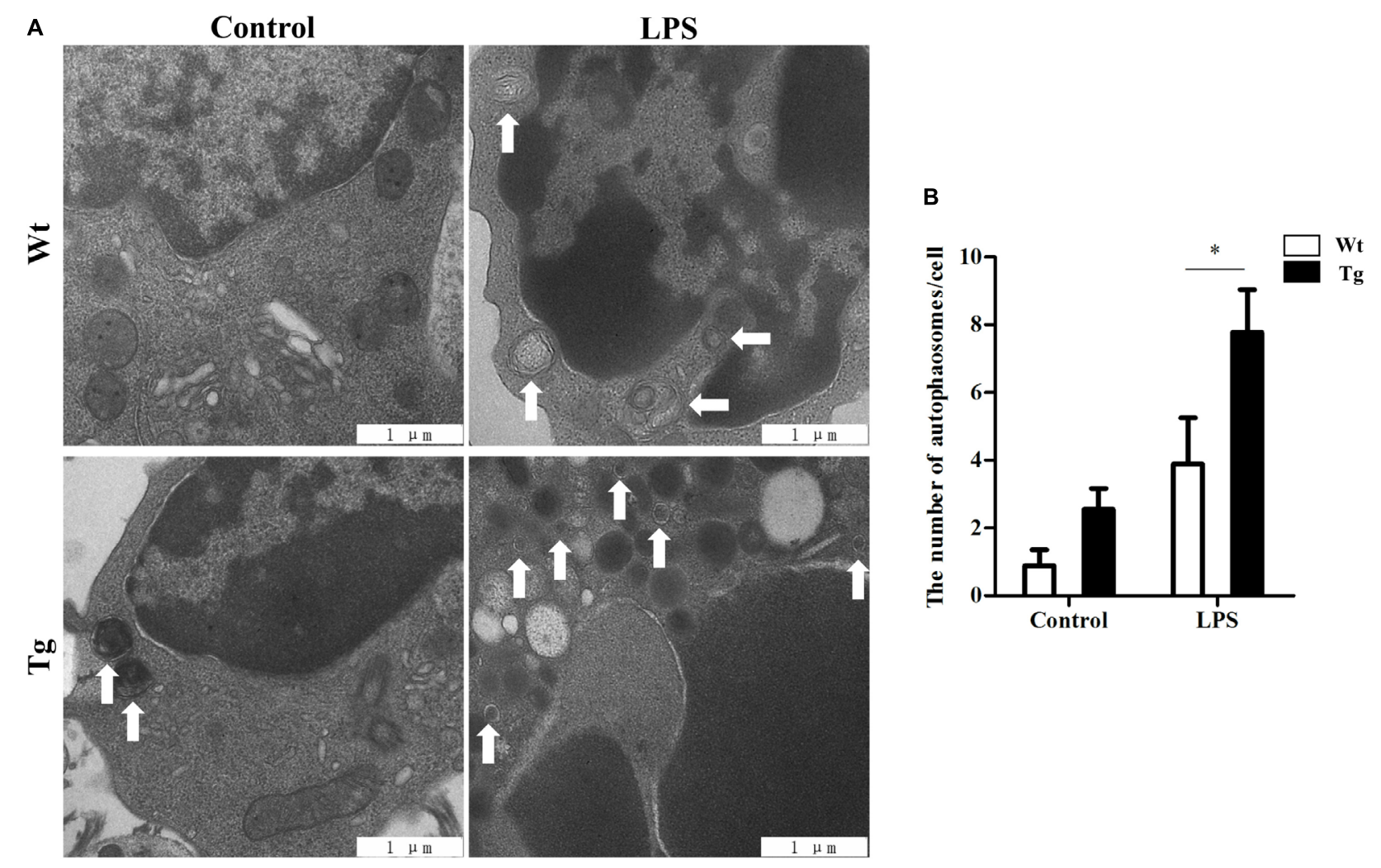

FIGURE 2 | Autophagosomes/autolysosomes increase in Tg sheep. (A) Transmission electron microscopy images: the white arrows indicate autophagosomes/autolysosomes; ×20,000; scale bar: $1 \mu \mathrm{m}$. (B) Statistical analysis of the number of autophagosomes/autolysosomes per cell. Statistical analysis of not fewer than 25 cells. Wt, wild-type sheep; Tg, transgenic sheep. All data are presented as the mean \pm SEM from three experiments. ${ }^{*} P<0.05$ vs. Wt group.

cells were dehydrated in a graded series of ethanol and embedded in epoxyresin. Ultrathin sections were observed under an $\mathrm{H}-7650$ microscope at $100 \mathrm{kV}$ (Hitachi).

\section{Laser Scanning Confocal Microscopy}

The monocytes were treated with LPS (100 ng/ml) (Sigma, L6529) for $12 \mathrm{~h}$, and then the cells were collected to measure the autophagy level by transmission electron microscopy. In inhibitory experiments, the cells were pretreated with $10 \mathrm{mM}$ 3-MA for $6 \mathrm{~h}$, and LPS (100 ng/ml) was added for another $12 \mathrm{~h}$. Then, the cells were fixed in $4 \%$ paraformaldehyde for $20 \mathrm{~min}$. The fixed cells were permeabilized by $0.3 \%$ Triton X100 for $10 \mathrm{~min}$. The cells were incubated with primary antibodies against LC3B (1:1,000, Abcam, ab51520) at $4^{\circ} \mathrm{C}$ for $12 \mathrm{~h}$ and then incubated with Cy3-labeled secondary antibody (1:500; Beyotime, A0516) and hoechst33342 (Solarbio). The level of LC3B was visualized using a laser scanning confocal microscope (Leica SP8) and a $\times 63$ oil objective.

\section{Detection of Intracellular ROS}

LPS-induced intracellular ROS was measured by adding dichlorodihydrofluorescein diacetate (DCFH-DA) at different time points $(0,2,8$, and $24 \mathrm{~h})$. Intracellular $\mathrm{O}^{2-}$ and $\mathrm{H}_{2} \mathrm{O}_{2}$ production was monitored by testing the fluorescence caused by oxidative DCF, and the green fluorescence signal indicates the ROS level. After the cells were treated with LPS, $10 \mu \mathrm{M}$ of DCFH-DA was added to the cells for $30 \mathrm{~min}$ at $37^{\circ} \mathrm{C}$. The intracellular ROS level was visualized using a fluorescence microscope (Olympus, X71).

\section{Measurement of Oxidative Stress}

The monocytes were stimulated with LPS for $12 \mathrm{~h}$, and the culture supernatants were collected with a detection kit (Njjcbio) according to the manufacturer's instructions to examine the contents of $\mathrm{NO}$ and malondialdehyde (MDA) by spectrophotometry.

\section{Determination of the Signals of Autophagy and ROS in Monocytes}

To analyze the interactions between TLR4, autophagy, and ROS, several inhibitors were used, namely, Tak242 - a TLR4 inhibitor (Sigma, pretreated for $6 \mathrm{~h}$ ), 3-MA - an autophagy inhibitor (Sigma, pretreated for $6 \mathrm{~h}$ ), NAC - an antioxidant and a free radical scavenger (Sigma, pretreated for $2 \mathrm{~h}$ ), and SB203580 - a specific inhibitor of p38 MAPK (Sigma, pretreated for $1 \mathrm{~h}$ ). After treating the cells with these inhibitors, the cells were harvested for detection of autophagy and oxidative level, and the supernatants were collected to test the inflammatory cytokines. 

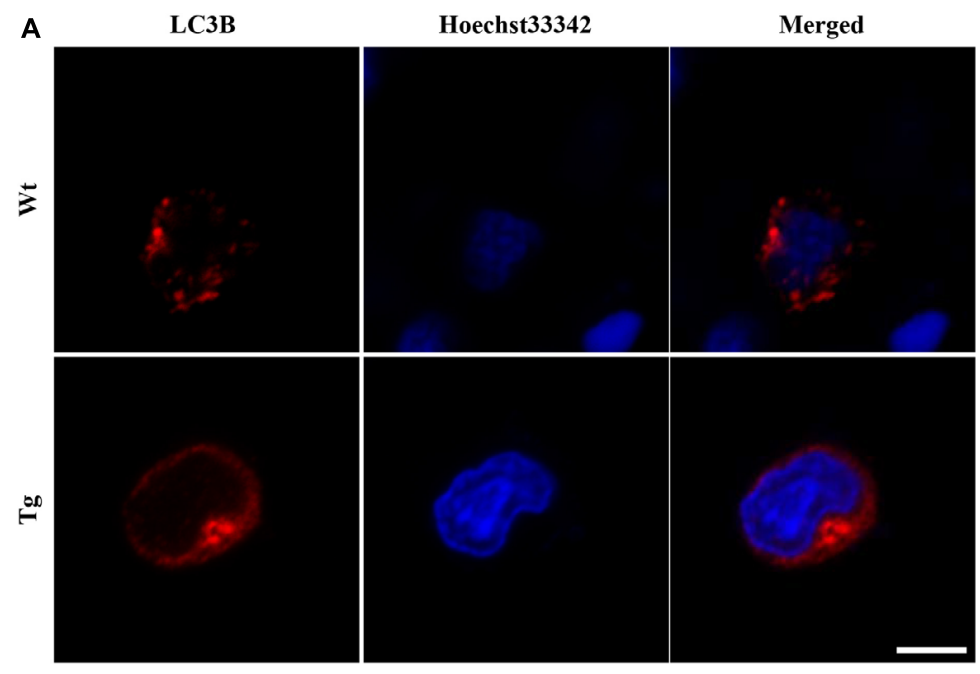

B

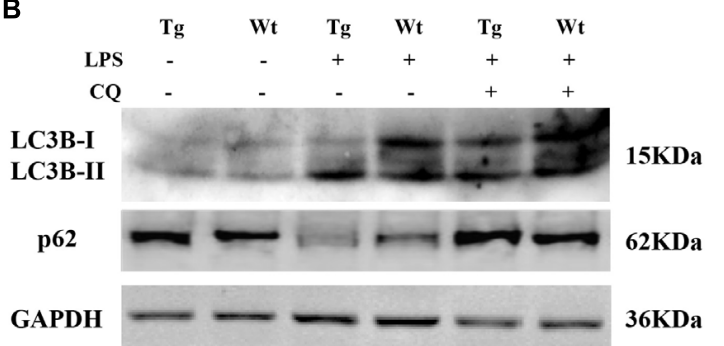

C

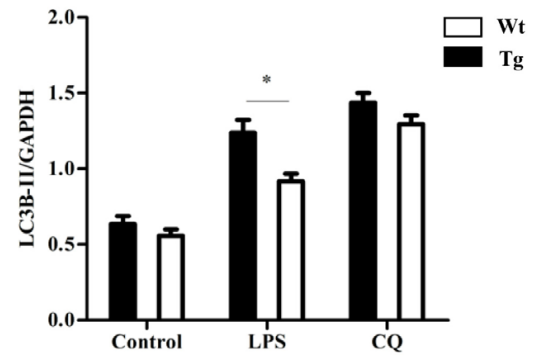

D

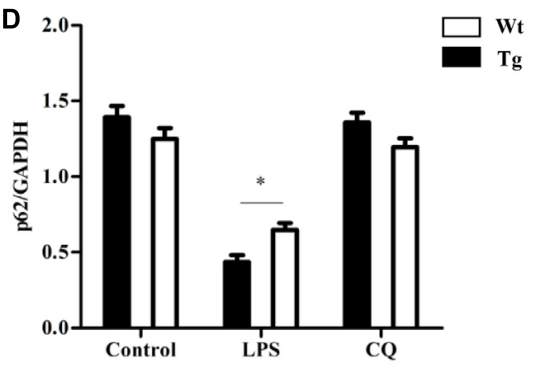

FIGURE 3 | LC3B increases in Tg sheep. (A) Immunofluorescence images of LC3B under laser scanning confocal microscope. Red: Cy3-labeled LC3B protein; blue, Hoechst33342-labeled nuclei; scale bar: $5 \mu \mathrm{m}$. (B-D) Analysis of LC3B and p62 level by Western blot. Wt, wild-type sheep; Tg, transgenic sheep. * $P<0.05$ vs. Wt group.

\section{ELISA Assay for Inflammatory Cytokines}

The monocytes were treated with LPS $(100 \mathrm{ng} / \mathrm{ml})$ for $12 \mathrm{~h}$, and then the cell culture supernatants were collected to measure the protein concentrations of IL-1 $\beta$, IL- 6 , IL-12, and TNF- $\alpha$ using ELISA kits (CUSABIO) according to the manufacturer's instructions.

\section{Injection of Lipopolysaccharide In vivo}

Six-month-old transgenic sheep and wild-type sheep $(n=3$ in each group) were subcutaneously injected with LPS $(0.04 \mathrm{mg} / \mathrm{ml}$ and $0.25 \mu \mathrm{g} / \mathrm{kg}$ ). At 0,2 , and $12 \mathrm{~h}$ after the LPS challenge, the serum was isolated for tests of proinflammatory cytokines and oxidative stress. Peripheral blood mononuclear cells (PBMCs) were collected for qRT-PCR. In qRT-PCR tests, GAPDH was chosen to normalize the data of each sample. Beclin-1, ATG5, WIPI1, and GAPDH primer sequences were as follows: Beclin-1: (F) 5'-GTCACCATCCAGGAGCTCACA- $3^{\prime}$ and (R) 5'-CACCATCCTGGCGAGTTTCA-3'; ATG5: (F) 5'-GCTTTAC TCCACTGCCGTCA-3' and (R) 5'-ACCAATGTTTCCAC TCCCTC-3'; WIPI1: (F) 5'-TCAGGAACCAGCGAAGAGA-3' and (R) 5'-ACGGCACGAAGTGATAGGA-3'; and GAPDH: (F) $5^{\prime}$-GTGTCTGTTGTGGATCTGACCTG-3' and (R) $5^{\prime}$ AGAAGAGTGAGTGTCGCTGTTGAAGT- $3^{\prime}$. The relative expression of mRNA was calculated by the $2^{-\Delta \Delta \mathrm{CT}}$ method.

\section{Statistical Analysis}

All data are shown as mean \pm SEM, and individual experiments were repeated not fewer than three times. Statistical analyses were performed by the univariate analysis of variance (ANOVA) followed by Student's $t$-test. $P<0.05$ was considered to be statistically significant.

\section{RESULTS}

\section{Screening of Tg Sheep Overexpressing TLR4}

The Ovis TLR4 CDS region was first cloned and linked to an expression vector that has a pCMV promoter (Figure 1A). Then, the linearized vector (digested with AseI) was transferred into the pronuclei of fertilized eggs by microinjection. The use of the restriction enzyme, HindIII, resulted in a 5,118 bp endogenous fragment present in all individual sheep and a 2,771 bp exogenous fragment that only exists in Tg sheep (Figure 1B). The relative TLR4 expression level in monocytes derived from peripheral blood was detected by qRT-PCR. The result indicated that the expression of TLR4 was significantly increased compared to the Wt control $\left({ }^{*} P<0.05\right)$ (Figure 1C). The Ovis TLR4 protein level 


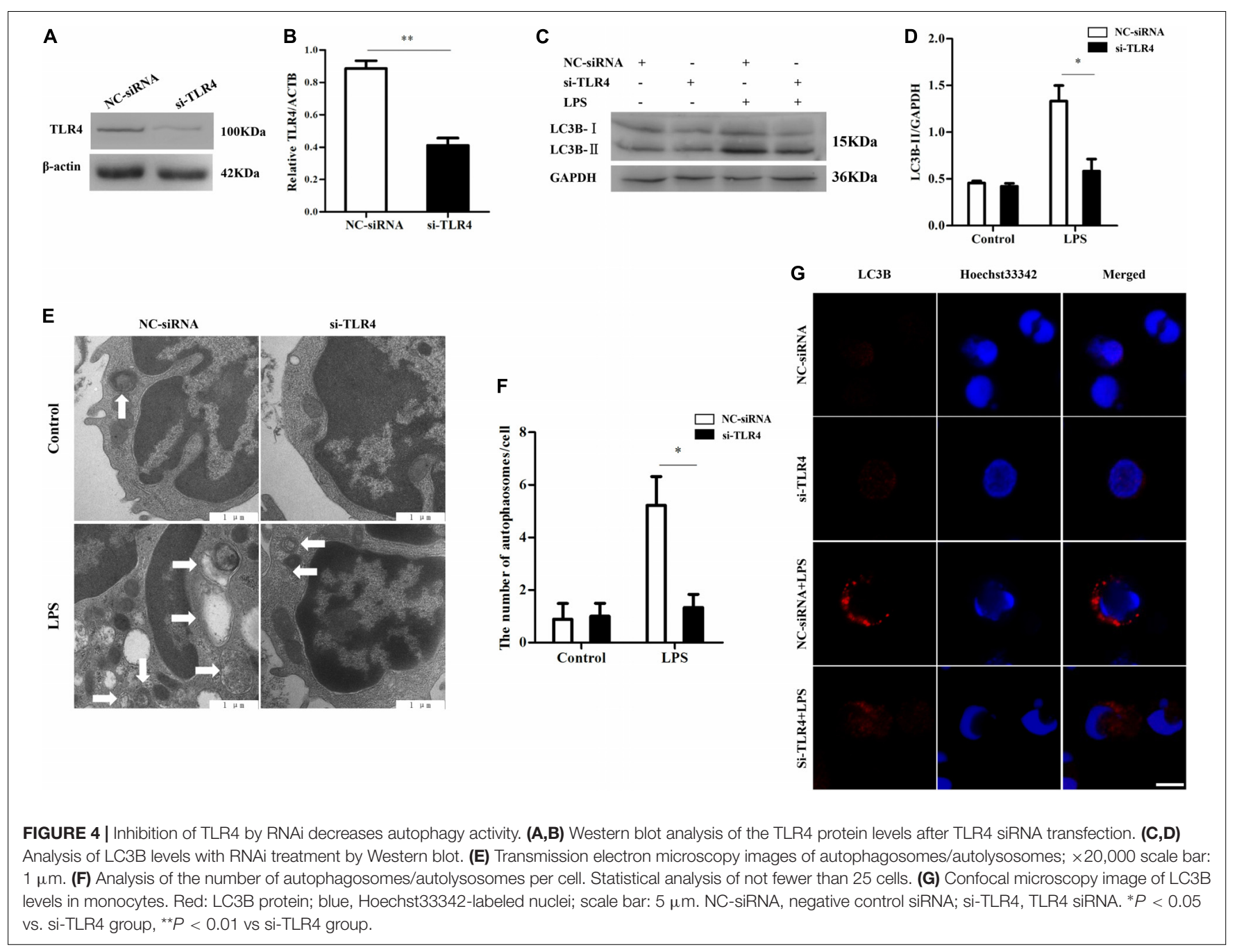

in $\mathrm{Tg}$ sheep was also higher than that in Wt control $\left({ }^{*} P<0.05\right)$ (Figures 1D,E).

\section{TLR4 Overexpression Increases Autophagy Activity}

To examine the autophagy activity in sheep peripheral blood monocytes, the autophagosomes were detected under transmission electron microscopy (TEM) (Figure 2). The autolysosomes in the TLR4-overexpression group were increased compared to those of the Wt group. After stimulating the cells with LPS (100 ng/ml for $12 \mathrm{~h}$ ), the number of autophagosomes in the Tg group was significantly higher than that in the $\mathrm{Wt}$ group $\left({ }^{*} P<0.05\right)$.

To further examine autophagy activity, the protein levels of the autophagosomal marker LC3B were detected by laser scanning confocal microscopy. LC3B protein was tagged by Cy3 (red), and the nuclei were tagged by hoechst33342 (blue). After stimulating the cells with LPS (100 ng/ml, for $12 \mathrm{~h}$ ), the images showed a distinctly increasing level of LC3B in the Tg group compared to the Wt group $\left({ }^{*} P<0.05\right)$ (scale bar: $5 \mu \mathrm{m}$; Figure $3 \mathrm{~A}$ ). In order to further determine whether the overexpression of TLR4 promotes the monocyte autophagy activity, p62 levels were also detected. LC3-II levels were up-regulated with LPS treatment and p62 levels were down-regulated. In addition, LPS-induced down-regulation of p62 could be reversed by chloroquine (CQ) treatment. After stimulating the cells with LPS, the results also showed an increasing level of LC3-II in the Tg group compared to the Wt group; meanwhile, the p62 levels in the Tg group was lower than that in the Wt group (Figures 3B-D). These results suggest that TLR4-overexpression increases the autophagy activity in sheep peripheral blood monocytes.

\section{Knock-Down of Ovis TLR4 Inhibits Autophagy Activity}

To further investigate the function of TLR4 during autophagy, RNAi was used to inhibit the expression of TLR4 in monocytes of sheep. Western blot results showed that the transfection of monocytes with si-TLR4 notably reduced the TLR4 protein level ${ }^{* *} P<0.01$ ) (Figures 4A,B). Furthermore, the down-regulation of TLR 4 by siRNA decreased the autophagosomes/autolysosomes and LC3B-II protein compared to the negative control siRNA (NC-siRNA) group $\left({ }^{*} P<0.05\right)$ (Figures $\left.4 \mathrm{C}-\mathrm{F}\right)$. In addition, 


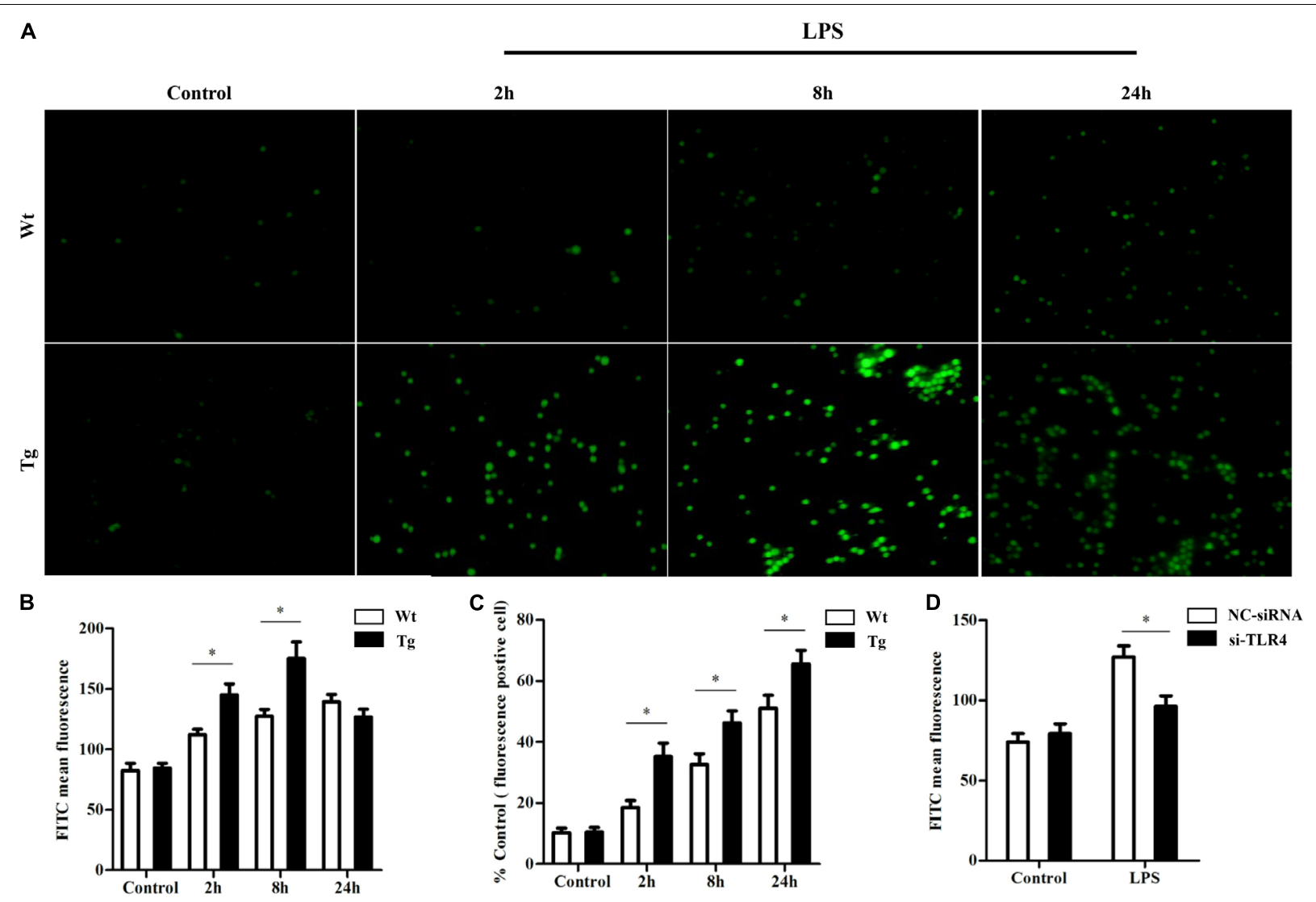

FIGURE 5 | TLR4 effects on reactive oxygen species (ROS) production by TLR4 overexpression or RNAi. (A,B) Analysis of ROS by DCFH-DA in monocytes. ROS was detected by dichlorodihydrofluorescein diacetate, and a fluorescence microscope was used to count the positive cells, $\times 200$. (C) ROS fluorescent positive cells; statistical analysis of not fewer than 100 cells. ${ }^{*} P<0.05$ vs. Wt group. (D) TLR4 RNAi effects on ROS production. ${ }^{*} P<0.05$ vs. NC-siRNA group.

A
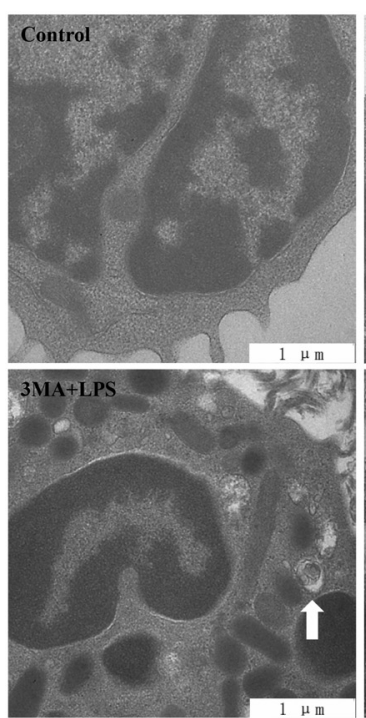
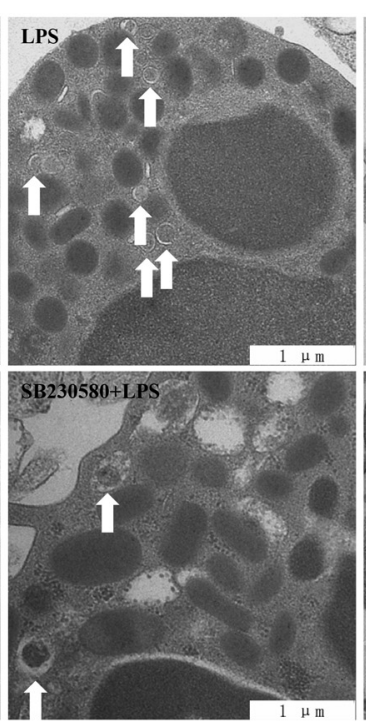

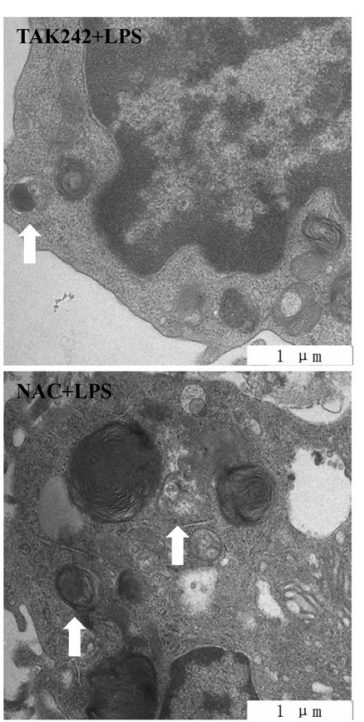

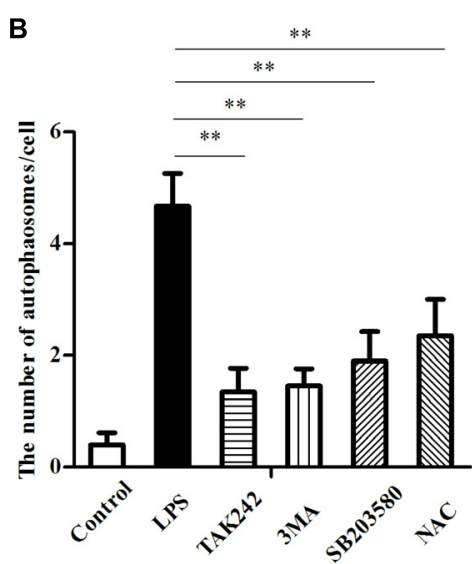

FIGURE 6 | TLR4-associated signaling is involved in the formation of autophagosomes/autolysosomes. (A) Cells were pretreated with either DMSO (0.1\%) or TAK242 (30 $\mu \mathrm{M})$ for $6 \mathrm{~h}$ or 3MA $(10 \mathrm{mM})$ for $6 \mathrm{~h}$, SB203580 $(5 \mu \mathrm{M})$ for $1 \mathrm{~h}$, or NAC $(1 \mathrm{mM})$ for $2 \mathrm{~h}$, and then the cells were treated with LPS (100 ng/ml) for another $6 \mathrm{~h}$. Transmission electron microscopy was used to survey the autophagy, $\times 20,000$; scale bar: $1 \mu \mathrm{m}$. (B) Statistical analysis of the number of autophagosomes per cell. Statistical analysis of not fewer than 25 cells. ${ }^{* *} P<0.01$ vs. LPS group. 


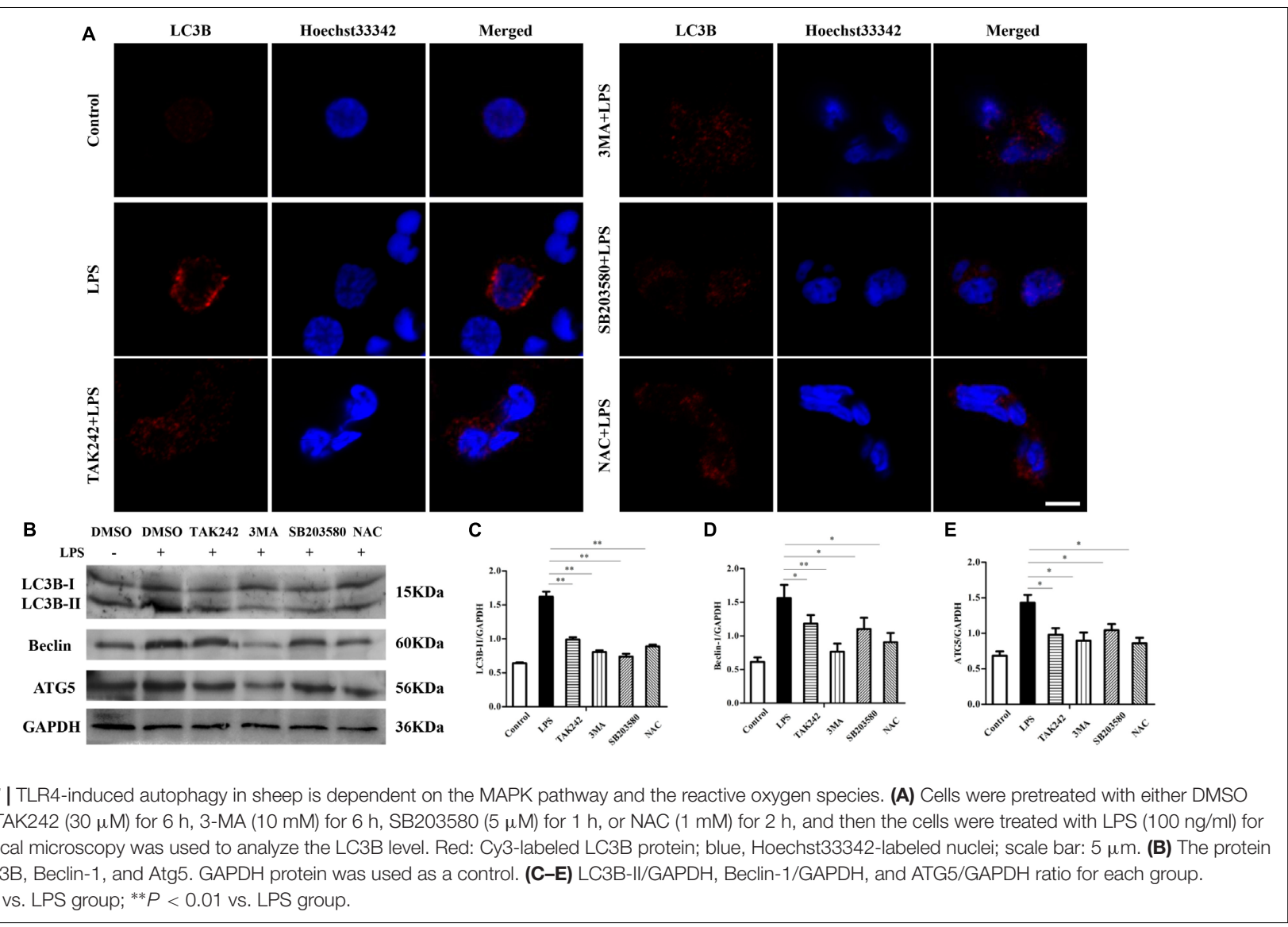

immunofluorescence staining showed that the LC3B level was significantly lower in the si-TLR4 group than in the NC-siRNA group $\left({ }^{*} P<0.05\right)$ (Figure $\left.4 \mathbf{G}\right)$. These results suggest that the down-regulation of TLR4 leads to a decrease in LPS-induced autophagy activity.

\section{Ovis TLR4 Effects on ROS Production}

As some studies have suggested that ROS production could induce autophagy and the above results showed that TLR4 influenced autophagy activity, we proceeded to explore whether TLR4 could affect ROS production. DCFH-DA staining showed that the overexpression of TLR4 promoted intracellular ROS production at an early stage $(2-8 \mathrm{~h})$, and then the ROS level quickly returned to normal values under LPS stress (Figures 5A-C). Furthermore, inhibition of TLR4 by si-TLR4 decreased the LPS-induced ROS (Figure 5D). These results suggest that TLR4 regulated the LPS-induced ROS production in sheep monocytes.

\section{TLR4 Activates the P38 MAPK Signaling Pathway and ROS Production to Promote LPS-Induced Autophagy}

Although in the data presented above there is a close relationship between TLR4 and LPS-induced autophagy, we still did not know what signaling pathway is involved. As LPS stimulation could activate TLR4-dependent p38 MAPK signaling and ROS, we next surveyed whether these players are involved in the autophagy process. As shown in Figure 6, the TLR4-specific inhibitor TAK242 (30 $\mu \mathrm{M}$, pretreated for $6 \mathrm{~h})$, the autophagy inhibitor 3MA (10 mM, pretreated for $6 \mathrm{~h})$, the MAPK-specific inhibitor SB203580 (5 $\mu \mathrm{M}$, pretreated for $1 \mathrm{~h}$ ), and the ROS inhibitor NAC ( $1 \mathrm{mM}$, pretreated for $2 \mathrm{~h}$ ) were used independently. The results showed the inhibition of TLR4, autophagy, MAPK, and ROS, and that all led to significantly decreased numbers of autophagosomes under TEM (Figures 6A,B) compared to the LPS group $\left({ }^{* *} P<0.01\right)$. Similarly, confocal results indicated that the LC3B protein level was also significantly reduced by TAK242, 3 -MA, SB203580, and NAC treatment $\left({ }^{*} P<0.05\right)$ (Figure 7A). Furthermore, beclin-1 and ATG5 are both autophagy-related genes. Western blot analysis showed that the inhibited TLR4, autophagy, MAPK signaling, and ROS decreased the protein levels of Beclin-1 and ATG5 $\left({ }^{*} P<0.05\right)$ (Figures 7B-E). In addition, inhibiting TLR4, autophagy, MAPK signaling, and ROS decreased the LC3B-II/GAPDH ratio $\left({ }^{*} P<0.05\right)$. These results suggest that p38 MAPK and ROS were both involved in the TLR4-dependent autophagy process.

\section{P38 MAPK Signaling and Autophagy Are Involved in Intracellular Oxidative Stress}

To further explore the relationship between TLR4, autophagy, and oxidation, different inhibitors were used. Figure 8 shows 
A
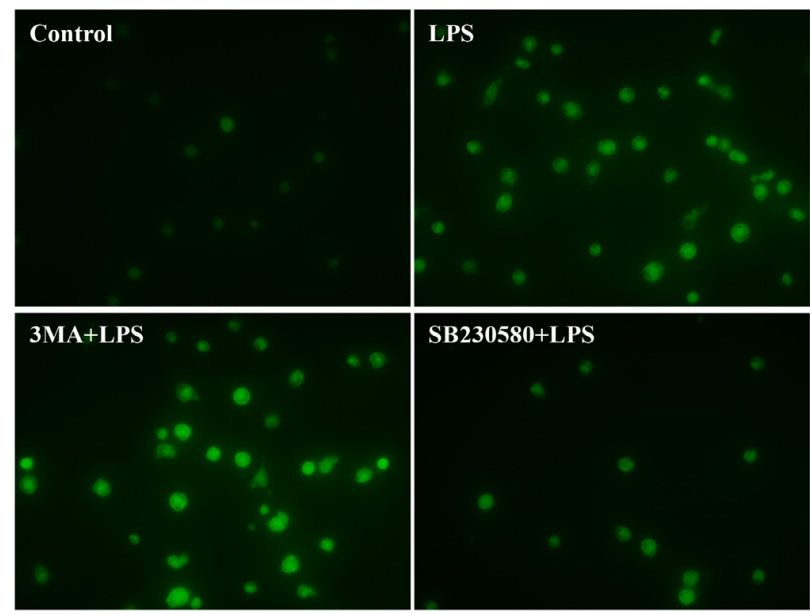

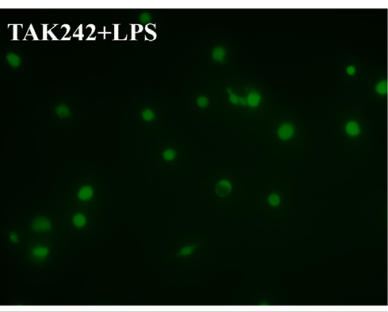

NAC + LPS

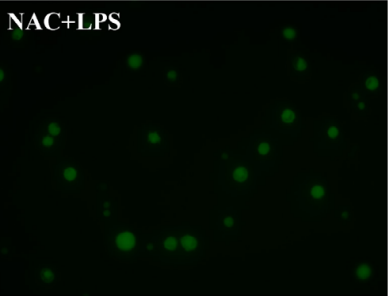

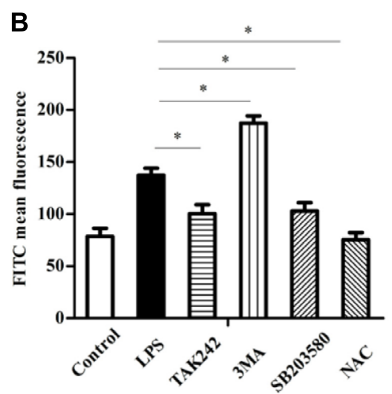
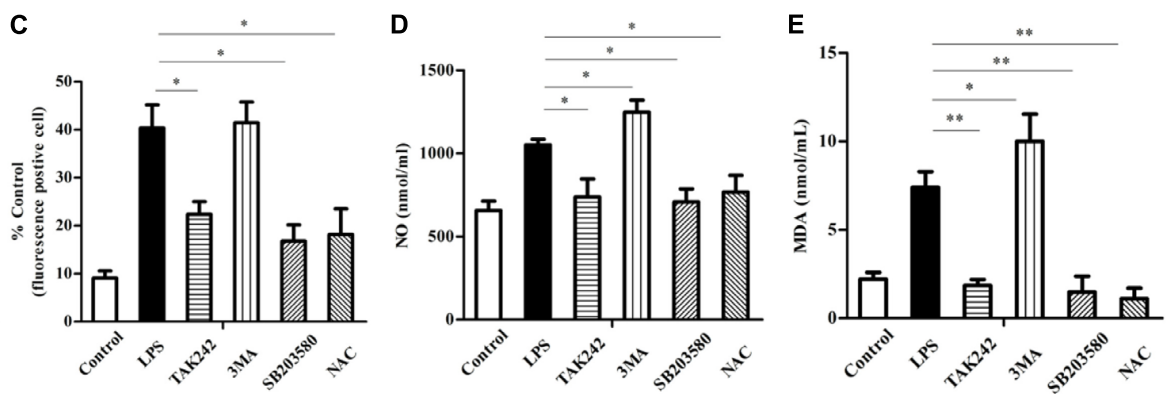

FIGURE 8 | TLR4-induced p38 MAPK signaling and autophagy effect on intracellular oxidative stress. (A-C) Analysis of reactive oxygen species (ROS) in sheep. ROS was detected by the DCFH-DA probe and a fluorescence microscope was used to count the positive cells, $\times 400$. (D) Level of NO. (E) Level of malondialdehyde. All data are presented as the mean \pm SEM from three experiments. ${ }^{*} P<0.05$ vs. LPS group; ${ }^{* *} P<0.01$ vs. LPS group.

that the inhibition of TLR4 decreased the LPS-induced ROS production and that the levels of $\mathrm{NO}$ and MDA also dropped $\left({ }^{*} P<0.05\right)$. After inhibiting autophagy by $3-\mathrm{MA}$, the ROS, NO, and MDA levels were up-regulated $\left({ }^{*} P<0.05\right)$ compared to the LPS group. In addition, after inhibiting P38 MAPK and ROS, the levels of ROS, NO, and MDA were all lower than those of the LPS group $\left({ }^{*} P<0.05\right)$. The above data suggest that TLR4-associated p38 MAPK signaling and autophagy were involved in regulating intracellular oxidative stress.

\section{The TLR4-Associated Signaling Pathway, Autophagy, and Oxidative Stress Influence Proinflammatory Cytokine Levels}

The LPS-induced activation of TLR4 often leads to the production of multiple cytokines to protect against infection. Figure 9 shows the inhibition of TLR4 decreased the protein levels of IL-1 $\beta$, IL-6, and TNF- $\alpha\left({ }^{*} P<0.05\right)$. Similarly, the inhibition of the intracellular oxidative stress down-regulated these proteins compared to the LPS group $\left({ }^{*} P<0.05\right)$. Blocking the P38 MAPK resulted in a dramatic drop of IL- $1 \beta$ and TNF- $\alpha$ $\left({ }^{*} P<0.05\right)$ but did not significantly reduce the IL-6 level, whereas 3 -MA markedly up-regulated the level of TNF- $\alpha\left({ }^{*} P<0.05\right)$. In addition, although 3 -MA increased IL- $1 \beta$ and IL-6, these differences were not statistically significant. Furthermore, all of these manipulations failed to alter the level of IL-12.

\section{TLR4 Participates in Oxidative Stress and Inflammatory Regulation by Promoting Autophagy in vivo}

To further explore the relationship between TLR4, autophagy, inflammation, and oxidative stress, in vivo studies were also performed. The results showed that the transgenic animals had a higher NO level at the early stage $(2 \mathrm{~h})$, and then the NO level quickly returned to normal values. The level of $\mathrm{NO}$ in transgenic sheep was markedly lower than that in the Wt group at $12 \mathrm{~h}$. The MDA level showed similar trends (Figures 10A,B). In addition, the proinflammatory cytokines IL-1 $\beta$, IL-6, and TNF$\alpha$ were significantly increased by LPS challenge in the Tg group at $2 \mathrm{~h}$. By $12 \mathrm{~h}$, the IL-1 $\beta$, IL-6, and TNF- $\alpha$ levels in the Tg group had dropped dramatically and were significantly lower than those in the Wt group (Figures 10C-E). Furthermore, qRT-PCR tests suggested that the relative mRNA expression of beclin-1, ATG5, and WIPI1 in PBMCs from the Tg group with LPS injection was higher than that in the Wt group at $12 \mathrm{~h}$ (Figures 10G-I). The autophagy machinery is initiated under stress conditions to maintain cellular homeostasis, and autophagy is generally thought to inhibit the excessive production of pro-inflammatory 


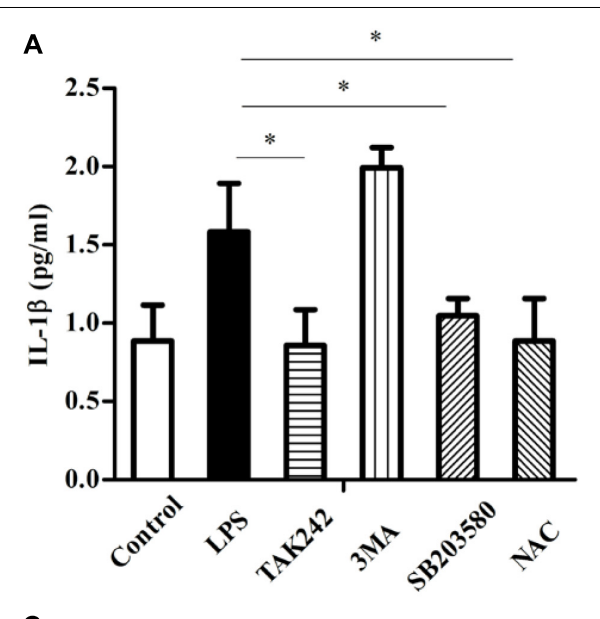

C

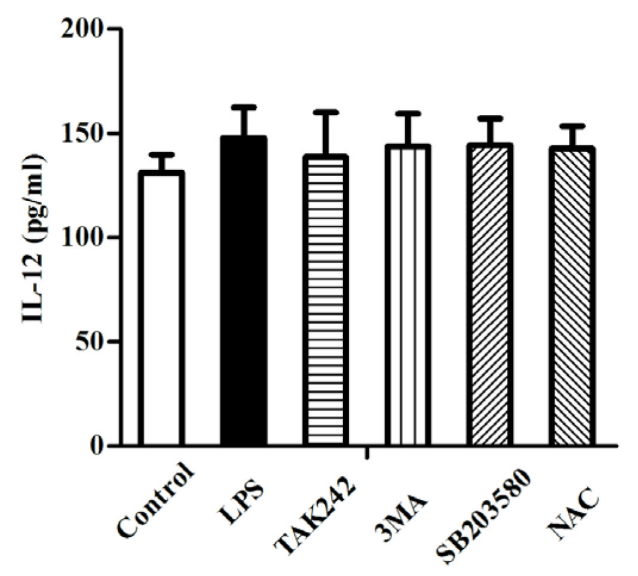

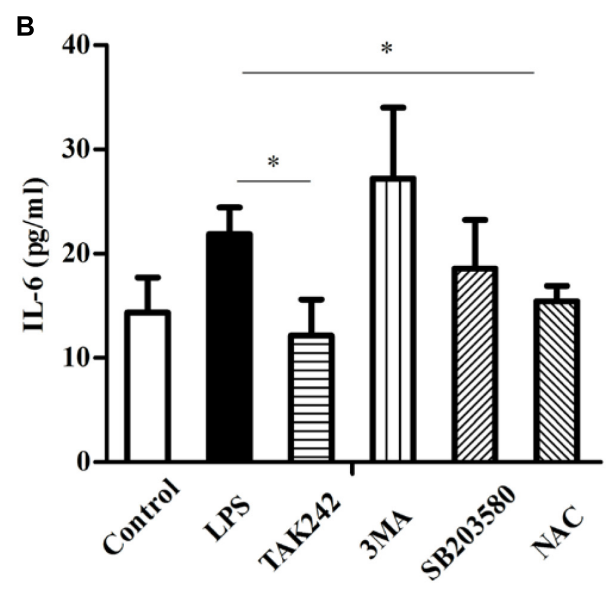

D

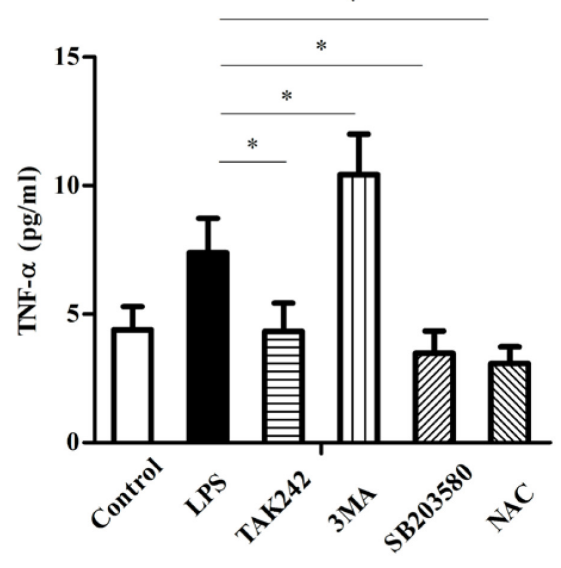

FIGURE 9 | TLR4-induced autophagy and reactive oxygen species effect on proinflammatory cytokine levels (ELISA) in sheep. (A) IL-1 $\beta$ levels. (B) IL-6 levels. (C) IL-12 levels. (D) TNF- $\alpha$ levels. All data are presented as the mean \pm SEM from three experiments. $* P<0.05$ vs LPS group.

cytokines and oxidative stress. These findings suggested that the high levels of TLR4 promoted the PBMC autophagy activity that suppressed the production of proinflammatory cytokines and oxidative stress.

\section{DISCUSSION}

PRRs mainly include TLRs, RIG-I-like receptors, and NOD-like receptors (NLRs) that can recognize most PAMPs. TLRs interact with the myeloid differentiation factor 88 (MyD88) to activate nuclear factor- $\kappa \mathrm{B}(\mathrm{NF}-\kappa \mathrm{B})$ and MAPKs, which subsequently activate activator protein 1 and induce the production of inflammatory cytokines (Kawai and Akira, 2010; DiDonato et al., 2012; O'Neill et al., 2013). TLR4 interacts with protein CD14 and myeloid differentiation factor 2 to form a complex that is endocytosed into endosomes rapidly (Zanoni et al., 2011). It has been reported that TLR4-mutant cells show lower sensitivity and susceptibility to LPS compared to wild-type cells (Faure et al., 2000; Jilling et al., 2006). In addition, the macrophages of TLR4-deficient mice cannot produce IL-1, IL6, and IL-12 cytokines under LPS stress (Haynes et al., 2001;
Seki et al., 2001). Moreover, mice overexpressing TLR4 showed improved disease resistance (Roy et al., 2006). Our previous study suggested that, compared to controls, the peripheral blood monocytes of Tg sheep overexpressing TLR4 secreted more proinflammatory cytokines, internalized more pathogens, and eliminated pathogens more quickly (Deng et al., 2012; Wang et al., 2018a,b). We also found that the overexpression of TLR4 promoted the activation of both NF- $\kappa \mathrm{B}$ and MAPK signaling at an early stage (0-4 h) (Wei et al., 2019). NF-кB and MAPK signaling pathways not only induced the production of pro-inflammatory cytokines but also trigger the release of oxidation intermediates (Ono and Han, 2000; Vladimirov, 2004). Thus, overexpression of TLR4 activated NF- $\kappa$ B and MAPK signaling and was responsible for the increased oxidative stress and production of pro-inflammatory cytokines. Our study showed that inhibiting TLR4 down-regulated the production of IL- $1 \beta$, IL- 6 and TNF- $\alpha$. Furthermore, inhibition of the p38 MAPK signals downstream of TLR4 decreased the secretion of IL- $1 \beta$ and TNF- $\alpha$.

Autophagy, as an essential biological pathway with effects on immunity, helps to eliminate intracellular pathogenic microorganisms. Autophagy controls inflammation by 


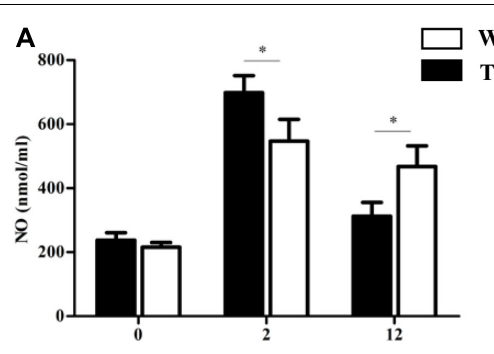

(h)

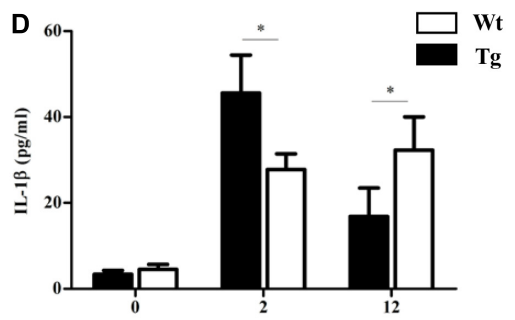

(h)

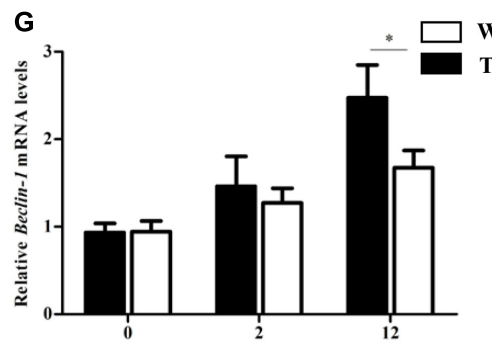

(h)

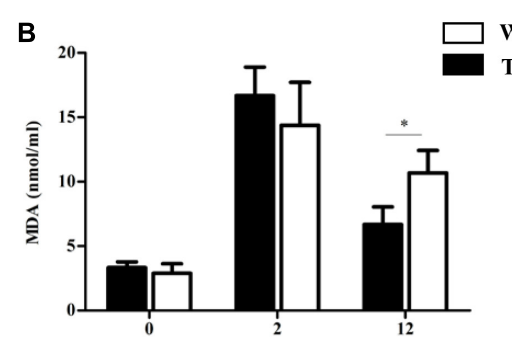

(h)

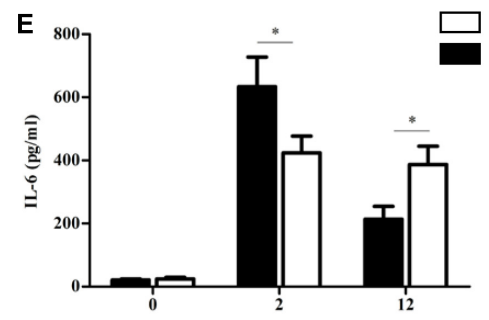

(h)

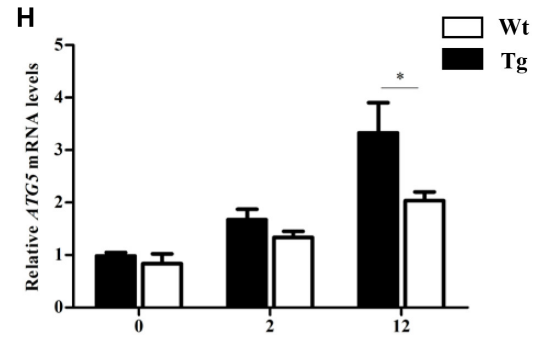

(h)

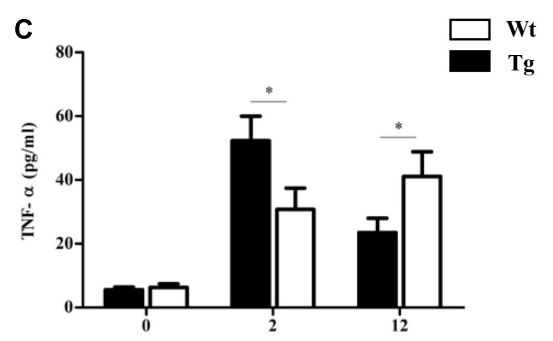

(h)

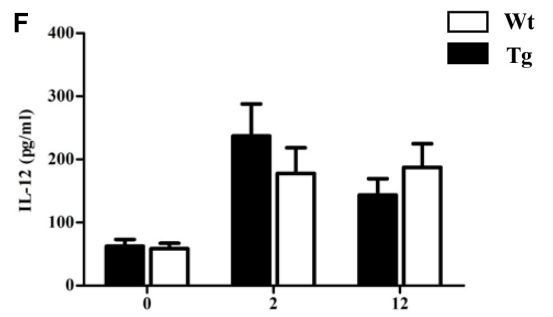

(h)

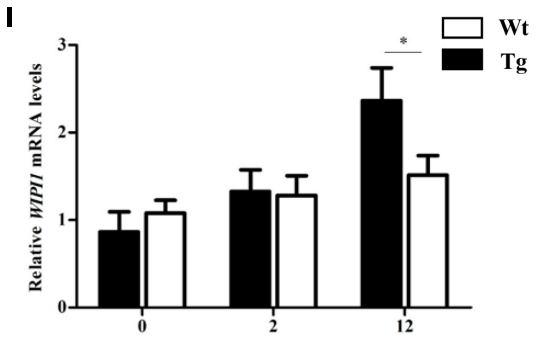

(h)

FIGURE 10 | The proinflammatory cytokines, NO and MDA levels, and PBMCs autophagy activity in sheep with LPS treatment in vivo. (A) NO levels.

(B) Malondialdehyde levels. (C) TNF- $\alpha$ levels. (D) IL-1 $\beta$ levels. (E) IL-6 levels. (F) IL-12 levels. (G-I) qRT-PCR analysis of Beclin-1, ATG5, and WIPI1 expression. All data are presented as the mean \pm SEM from three experiments. ${ }^{*} P<0.05$ vs. Wt group.

interacting with the regulation of the innate immune signals, removing endogenous inflammasome agonists, and affecting immune mediators. Generally, the inhibitors of nuclear factor kappa-B kinase, TAB2/3, and TAK control autophagy together. After PAMP induction, the dissociation of TAB2/3 from Beclin-1 leads to autophagy activation and autophagosome formation (Deretic et al., 2013). The ubiquitination of Beclin-1 by TRAF6 is mediated by TLR4-MyD88 signaling, and then TRAF6 dissociates BCL-2 from Beclin-1. Moreover, it has been reported that TLR4-induced TICAM1 was essential for the activation of autophagy (Zhan et al., 2014). However, cytokine IL-1 $\beta$ may be involved in TRAF 6-dependent Beclin-1 ubiquitination through MyD88 in macrophages (Nakahira et al., 2011; Zhou et al., 2011). A recent report showed that rapamycin (autophagy enhancer) could potently suppress the production of IL- $1 \beta$, IL- 6 , TNF- $\alpha$, and NO in BV2 cells. Furthermore, it has been reported that NF$\kappa \mathrm{B}$ also communicated the connection between autophagy and inflammasome activation. NF- $\kappa \mathrm{B}$ promoted the elimination of damaged organelles by the activation of the autophagy receptor SQSTM1 and inhibited IL-1 $\beta$ secretion via NLRP3 signals (Zhong et al., 2016). Recently, it was shown that p38 MAPK participates in the production of inflammatory cytokines (Du et al., 2018). These studies suggested that autophagy is closely related to TLR4 and the inflammatory factors. In this study, TLR4 and its downstream signaling p38 MAPK affected the autophagy in sheep monocytes. We found that the overexpression of TLR4 increased the LPS-induced number of autophagosome in monocytes and that the LC3B-II/GAPDH ratio was also increased (Figures 2, 3). Our research further suggests that the down-regulation of TLR4 activity by RNAi (Figure 4) or the TAK242 inhibitor (Figures 6, 7) both decreased the intracellular autophagosomes and the ratio of LC3B-II/GAPDH. In addition, the results also showed that the protein levels of Beclin-1 and ATG5 were down-regulated after TLR4 or p38 MAPK inhibition in Tg peripheral blood monocytes (Figures 6, 7). Previous experiments have mostly focused on the effects of the TLR4-TRAF6 pathway on autophagy (Shi and Kehrl, 2010; Nazio et al., 2013; Zhan et al., 2014). In our Tg animal model, we found that the TLR4-p38 MAPK pathway is also involved in autophagy. The inhibition of TLR4 also decreases the levels of IL- $1 \beta$, IL- 6 , and TNF- $\alpha$, while blocking the p38 MAPK pathway using SB203580 decreases the secretion of IL-1 $\beta$ and TNF- $\alpha$ (Figure 9).

Evidence showed that ROS production is related to TLR4 activation and autophagy (Shin et al., 2013). It has been reported that NF- $\mathrm{B}$ and p38 MAPK signaling involves interactions 
between autophagy and ROS (He et al., 2013; Zhu et al., 2017). The MAPK pathway mediated by TLR4 under LPS stress participates in cell survival with autophagy (Kim et al., 2013). However, the production of intracellular ROS also plays an essential role in the induction of MAPK, and evidence suggested that ROS could induce MAPK signaling (Bui-Xuan et al., 2010). Moreover, our previous research showed that the MAPK signal is involved in TLR4-mediated bacterial internalization which is mainly about phagocytosis (Wang et al., 2018a). In the current study, the ROS level in sheep over-expressing TLR4 was markedly higher than that in the wild-type group, and it returned to normal by knocking-down TLR4 (Figure 5). Moreover, the suppression of autophagy with 3-MA promoted ROS production and significantly increased the level of NO and MDA (Figure 8). Additionally, the inactivation of p38 MAPK decreased the LPS-induced ROS by 38 MAPK-specific inhibitor SB203580. Similarly, the inactivation of p38 MAPK signals downregulated the expression of NO and MDA (Figure 8). Evidence suggested that pathogenic Gram-negative bacteria would first stimulate inflammatory secretion and generate ROS which are mostly produced by NOX (Huang et al., 2009). ROS could activate iNOS via NF-кB and lead to the release of NO which helped to eliminate pathogenic bacteria by producing peroxidase and superoxide (Heo et al., 2008). The excess oxide would cause organelle damage, but ROS also activates autophagy, which helps to clean the damaged organelles and maintain intracellular homeostasis. Our data in Figure 5B showed that, in the Tg animal, the ROS level increased rapidly at an early stage (peak at $8 \mathrm{~h}$ ) and then began to fall. Even though the exact underlying mechanisms are unknown, these dynamics may be caused by a high level of autophagy in the $\mathrm{Tg}$ group. Taking these data together, under LPS stress, TLR4 induces ROS production to mediate autophagy via the p38 MAPK signaling pathway.

To further explore the relationship between TLR4, autophagy, inflammation, and oxidative stress in vivo, the transgenic sheep and Wt counterparts were treated with LPS. We found that the serum proinflammatory cytokines levels and oxidative stress in transgenic sheep increased rapidly at an early stage and then began to fall. By $12 \mathrm{~h}$ after the LPS challenge, the NO, MDA, and proinflammatory cytokine levels in the transgenic sheep were markedly lower than those in the Wt group. WIPI1, Beclin-1, and ATG5 were selected as the indices of autophagy. Because of its reliability, WIPI1 can be used as a marker of autophagy activity by qRT-PCR analysis (Tsuyuki et al., 2014; Proikas-Cezanne et al., 2015; Tao et al., 2018). In vivo experiments show that the autophagy activity of PBMCs increased gradually with LPS treatment, and the autophagy activity in the PBMCs of transgenic sheep was significantly higher than that in the Wt sheep at $12 \mathrm{~h}$ after the LPS challenge. These findings in vivo were similar to those of the in vitro experiments. Cytokines and oxidative stress orchestrate the initiation, development, and recovery during the disease process, and autophagy plays a key role under various stress conditions to maintain cellular homeostasis (Netea-Maier et al., 2016). Several reports showed that TLR4 participated in the regulation of autophagy (Yu et al., 2017;
Li et al., 2019). Our previous study found that the overexpression of TLR4 promoted the activation of both NF- $\kappa B$ and MAPK signaling (Wei et al., 2019). In this study, we found that p38 MAPK signaling pathway and ROS production were involved in TLR4-mediated autophagy. Evidence also showed that the autophagy process suppressed all kinds of proinflammatory cytokines and oxidative stress (Kim et al., 2017; Han et al., 2018; Monkkonen and Debnath, 2018). AKT/mTOR signaling is involved in the induction of autophagy to attenuate LPSinduced proinflammatory cytokines. Furthermore, autophagydeficient ovarian cancer cells display increased ROS and a decrease in autophagy due to the knock-down of BECN1, leading to the up-regulation of ROS and NF- $\kappa$ B activation (Qin et al., 2015; Zhao et al., 2016). So, we thought that the high levels of TLR4 promoted the PBMC autophagy activity that suppressed the production of proinflammatory cytokines and oxidative stress.

In summary, in our current research based on a transgenic sheep model over-expressing TLR4, we surveyed the relationship between ROS, the inflammatory response, and autophagy. The results suggest that transgenic animals have a stronger autophagy activity than the wild-type counterparts in vitro and in vivo. Furthermore, the autophagy in sheep was mediated by TLRinduced ROS via p38 MAPK signaling. Herein we provided a novel animal model to study the effects of TLR4 on autophagy, inflammation, and oxidative stress. In addition, this study also provides valuable insights into Gram-negative bacteriosis.

\section{DATA AVAILABILITY STATEMENT}

All data underlying the findings are included in the article and fully available without restriction.

\section{ETHICS STATEMENT}

The animal study was reviewed and approved by the Animal Welfare Committee of the Northeast Agricultural University.

\section{AUTHOR CONTRIBUTIONS}

Conceptualization was by SW and SD. Data curation and Formal analysis was by SW and XS. Funding acquisition was by ZL and YY. Methodology was by SW. Writing and discussion were carried out by SW, KZ, PJ, MQ, and YY.

\section{FUNDING}

This research was supported by grants from the National Transgenic Creature Breeding Grand Project (2016ZX08008003), Special fund for scientific innovation strategy-construction of high level Academy of Agriculture Science (R2019YJYB2004), and the Guangzhou Science and Technology planning project (201707020007). 


\section{REFERENCES}

Bui-Xuan, N. H., Tang, P. M., Wong, C. K., and Fung, K. P. (2010). Photo-activated pheophorbide-a, an active component of Scutellaria barbata, enhances apoptosis via the suppression of ERK-mediated autophagy in the estrogen receptor-negative human breast adenocarcinoma cells MDA-MB-231. J. Ethnopharmacol. 131, 95-103. doi: 10.1016/j.jep.2010.06.007

Chen, G., Shaw, M. H., Kim, Y. G., and Nunez, G. (2009). NOD-like receptors: role in innate immunity and inflammatory disease. Annu. Rev. Pathol. 4, 365-398. doi: 10.1146/annurev.pathol.4.110807.092239

Deng, S., Wu, Q., Yu, K., Zhang, Y., Yao, Y., Li, W., et al. (2012). Changes in the relative inflammatory responses in sheep cells overexpressing of toll-like receptor 4 when stimulated with LPS. PLoS One 7:e47118. doi: 10.1371/journal. pone.0047118

Deng, S., Yu, K., Zhang, B., Yao, Y., Wang, Z., Zhang, J., et al. (2015). Toll-Like receptor 4 promotes no synthesis by upregulating GCHI expression under oxidative stress conditions in sheep monocytes/macrophages. Oxid. Med. Cell Longev. 2015:359315. doi: 10.1155/2015/359315

Deretic, V., Saitoh, T., and Akira, S. (2013). Autophagy in infection, inflammation and immunity. Nat. Rev. Immunol. 13, 722-737. doi: 10.1038/nri3532

DiDonato, J. A., Mercurio, F., and Karin, M. (2012). NF-kappaB and the link between inflammation and cancer. Immunol. Rev. 246, 379-400. doi: 10.1111/j. 1600-065X.2012.01099.x

Du, K., Zhou, M., Li, Q., and Liu, X. Z. (2018). Chlamydia trachomatis inhibits the production of pro-inflammatory cytokines in human PBMCs through induction of IL-10. J. Med. Microbiol. 67, 240-248. doi: 10.1099/jmm.0.000672

Faure, E., Equils, O., Sieling, P. A., Thomas, L., Zhang, F. X., Kirschning, C. J., et al. (2000). Bacterial lipopolysaccharide activates NF-kappaB through tolllike receptor 4 (TLR-4) in cultured human dermal endothelial cells. Differential expression of TLR-4 and TLR-2 in endothelial cells. J. Biol. Chem. 275, 1105811063. doi: 10.1074/jbc.275.15.11058

Han, F., Xiao, Q. Q., Peng, S., Che, X. Y., Jiang, L. S., Shao, Q., et al. (2018). Atorvastatin ameliorates LPS-induced inflammatory response by autophagy via AKT/mTOR signaling pathway. J. Cell. Biochem. 119, 1604-1615. doi: 10.1002/ jcb. 26320

Haynes, L. M., Moore, D. D., Kurt-Jones, E. A., Finberg, R. W., Anderson, L. J., and Tripp, R. A. (2001). Involvement of toll-like receptor 4 in innate immunity to respiratory syncytial virus. J. Virol. 75, 10730-10737. doi: 10.1128/JVI.75.22. 10730-10737.2001

He, H., Zang, L. H., Feng, Y. S., Chen, L. X., Kang, N., Tashiro, S., et al. (2013). Physalin A induces apoptosis via p53-Noxa-mediated ROS generation, and autophagy plays a protective role against apoptosis through p38-NF-kappaB survival pathway in A375-S2 cells. J. Ethnopharmacol. 148, 544-555. doi: 10. 1016/j.jep.2013.04.051

Heo, S. K., Yun, H. J., Noh, E. K., Park, W. H., and Park, S. D. (2008). LPS induces inflammatory responses in human aortic vascular smooth muscle cells via Tolllike receptor 4 expression and nitric oxide production. Immunol. Lett. 120, 57-64. doi: 10.1016/j.imlet.2008.07.002

Hoareau, L., Bencharif, K., Rondeau, P., Murumalla, R., Ravanan, P., Tallet, F., et al. (2010). Signaling pathways involved in LPS induced TNFalpha production in human adipocytes. J. Inflamm. 7:1. doi: 10.1186/1476-9255-7-1

Huang, J., Canadien, V., Lam, G. Y., Steinberg, B. E., Dinauer, M. C., Magalhaes, M. A., et al. (2009). Activation of antibacterial autophagy by NADPH oxidases. Proc. Natl. Acad. Sci. U.S.A. 106, 6226-6231. doi: 10.1073/pnas.081104 5106

Janeway, C. A. Jr., and Medzhitov, R. (2002). Innate immune recognition. Annu. Rev. Immunol. 20, 197-216. doi: 10.1146/annurev.immunol.20.083001.084359

Jilling, T., Simon, D., Lu, J., Meng, F. J., Li, D., Schy, R., et al. (2006). The roles of bacteria and TLR4 in rat and murine models of necrotizing enterocolitis. J. Immunol. 177, 3273-3282. doi: 10.4049/jimmunol.177.5.3273

Kawai, T., and Akira, S. (2010). The role of pattern-recognition receptors in innate immunity: update on Toll-like receptors. Nat. Immunol. 11, 373-384. doi: $10.1038 /$ ni.1863

Kim, G. D., Oh, J., Park, H. J., Bae, K., and Lee, S. K. (2013). Magnolol inhibits angiogenesis by regulating ROS-mediated apoptosis and the PI3K/AKT/mTOR signaling pathway in mES/EB-derived endothelial-like cells. Int. J. Oncol. 43, 600-610. doi: 10.3892/ijo.2013.1959
Kim, K. Y., Park, K. I., Kim, S. H., Yu, S. N., Park, S. G., Kim, Y. W., et al. (2017). Inhibition of autophagy promotes salinomycin-induced apoptosis via reactive oxygen species-mediated PI3K/AKT/mTOR and ERK/p38 MAPK-Dependent signaling in human prostate cancer cells. Int. J. Mol. Sci. 18:1088. doi: 10.3390/ ijms 18051088

Li, J., Li, B., Cheng, Y., Meng, Q., Wei, L., Li, W., et al. (2019). The synergistic effect of NOD2 and TLR4 on the activation of autophagy in human submandibular gland inflammation. J. Oral Pathol. Med. 48, 87-95. doi: 10.1111/jop.12793

Monkkonen, T., and Debnath, J. (2018). Inflammatory signaling cascades and autophagy in cancer. Autophagy 14, 190-198. doi: 10.1080/15548627.2017. 1345412

Nakahira, K., Haspel, J. A., Rathinam, V. A., Lee, S. J., Dolinay, T., Lam, H. C., et al. (2011). Autophagy proteins regulate innate immune responses by inhibiting the release of mitochondrial DNA mediated by the NALP3 inflammasome. Nat. Immunol. 12, 222-230. doi: 10.1038/ni.1980

Nazio, F., Strappazzon, F., Antonioli, M., Bielli, P., Cianfanelli, V., Bordi, M., et al. (2013). mTOR inhibits autophagy by controlling ULK1 ubiquitylation, selfassociation and function through AMBRA1 and TRAF6. Nat. Cell Biol. 15, 406-416. doi: 10.1038/ncb2708

Netea-Maier, R. T., Plantinga, T. S., van de Veerdonk, F. L., Smit, J. W., and Netea, M. G. (2016). Modulation of inflammation by autophagy: consequences for human disease. Autophagy 12, 245-260. doi: 10.1080/15548627.2015.1071759

O’Neill, L. A., Golenbock, D., and Bowie, A. G. (2013). The history of Toll-like receptors - redefining innate immunity. Nat. Rev. Immunol. 13, 453-460. doi: $10.1038 /$ nri3446

Ono, K., and Han, J. (2000). The p38 signal transduction pathway: activation and function. Cell. Signal. 12, 1-13. doi: 10.1016/s0898-6568(99)00071-76

Pacquelet, S., Johnson, J. L., Ellis, B. A., Brzezinska, A. A., Lane, W. S., Munafo, D. B., et al. (2007). Cross-talk between IRAK-4 and the NADPH oxidase. Biochem. J. 403, 451-461. doi: 10.1042/BJ20061184

Park, H. S., Chun, J. N., Jung, H. Y., Choi, C., and Bae, Y. S. (2006). Role of NADPH oxidase 4 in lipopolysaccharide-induced proinflammatory responses by human aortic endothelial cells. Cardiovasc. Res. 72, 447-455. doi: 10.1016/j.cardiores. 2006.09.012

Proikas-Cezanne, T., Takacs, Z., Donnes, P., and Kohlbacher, O. (2015). WIPI proteins: essential PtdIns3P effectors at the nascent autophagosome. J. Cell Sci. 128, 207-217. doi: 10.1242/jcs. 146258

Qin, W., Li, C., Zheng, W., Guo, Q., Zhang, Y., Kang, M., et al. (2015). Inhibition of autophagy promotes metastasis and glycolysis by inducing ROS in gastric cancer cells. Oncotarget 6, 39839-39854. doi: 10.18632/oncotarget.5674

Roy, M. F., Lariviere, L., Wilkinson, R., Tam, M., Stevenson, M. M., and Malo, D. (2006). Incremental expression of Tlr4 correlates with mouse resistance to Salmonella infection and fine regulation of relevant immune genes. Genes Immun. 7, 372-383. doi: 10.1038/sj.gene.6364309

Ryan, K. A., Smith, M. F. Jr., Sanders, M. K., and Ernst, P. B. (2004). Reactive oxygen and nitrogen species differentially regulate Toll-like receptor 4-mediated activation of NF-kappa B and interleukin-8 expression. Infect. Immun. 72, 2123-2130. doi: 10.1128/iai.72.4.2123-2130.2004

Sciarretta, S., Volpe, M., and Sadoshima, J. (2014). NOX4 regulates autophagy during energy deprivation. Autophagy 10, 699-701. doi: 10.4161/auto.27955

Seki, E., Tsutsui, H., Nakano, H., Tsuji, N., Hoshino, K., Adachi, O., et al. (2001). Lipopolysaccharide-induced IL-18 secretion from murine Kupffer cells independently of myeloid differentiation factor 88 that is critically involved in induction of production of IL-12 and IL-1beta. J. Immunol. 166, 2651-2657. doi: 10.4049/jimmunol.166.4.2651

Shi, C. S., and Kehrl, J. H. (2010). TRAF6 and A20 regulate lysine 63-linked ubiquitination of Beclin-1 to control TLR4-induced autophagy. Sci. Signal. 3:ra42. doi: 10.1126/scisignal.2000751

Shin, S., Jing, K., Jeong, S., Kim, N., Song, K. S., Heo, J. Y., et al. (2013). The omega-3 polyunsaturated fatty acid DHA induces simultaneous apoptosis and autophagy via mitochondrial ROS-mediated Akt-mTOR signaling in prostate cancer cells expressing mutant p53. Biomed. Res. Int. 2013:568671. doi: 10.1155/ 2013/568671

Singh, R., and Cuervo, A. M. (2011). Autophagy in the cellular energetic balance. Cell Metab. 13, 495-504. doi: 10.1016/j.cmet.2011.04.004

Suzuki, Y., Hattori, K., Hamanaka, J., Murase, T., Egashira, Y., Mishiro, K., et al. (2012). Pharmacological inhibition of TLR4-NOX4 signal protects against 
neuronal death in transient focal ischemia. Sci. Rep. 2:896. doi: 10.1038/ srep00896

Takeuchi, O., and Akira, S. (2010). Pattern recognition receptors and inflammation. Cell 140, 805-820. doi: 10.1016/j.cell.2010.01.022

Tao, J., Yang, M., Wu, H., Ma, T., He, C., Chai, M., et al. (2018). Effects of AANAT overexpression on the inflammatory responses and autophagy activity in the cellular and transgenic animal levels. Autophagy 14, 1850-1869. doi: 10.1080/ 15548627.2018.1490852

Tse, K. H., Chow, K. B., Leung, W. K., Wong, Y. H., and Wise, H. (2014). Lipopolysaccharide differentially modulates expression of cytokines and cyclooxygenases in dorsal root ganglion cells via Toll-like receptor-4 dependent pathways. Neuroscience 267, 241-251. doi: 10.1016/j.neuroscience.2014. 02.041

Tsuyuki, S., Takabayashi, M., Kawazu, M., Kudo, K., Watanabe, A., Nagata, Y., et al. (2014). Detection of WIPI1 mRNA as an indicator of autophagosome formation. Autophagy 10, 497-513. doi: 10.4161/auto.27419

Vladimirov, Y. A. (2004). Reactive oxygen and nitrogen species: diagnostic, preventive and therapeutic values. Biochemistry 69, 1-3. doi: 10.1023/b:biry. 0000016343.21774.c4

Wang, S., Cao, Y., Deng, S., Jiang, X., Wang, J., Zhang, X., et al. (2018a). Overexpression of toll-like receptor 4-linked mitogen-activated protein kinase signaling contributes to internalization of Escherichia coli in sheep. Int. J. Biol. Sci. 14, 1022-1032. doi: 10.7150/ijbs. 25275

Wang, S., Deng, S., Cao, Y., Zhang, R., Wang, Z., Jiang, X., et al. (2018b). Overexpression of toll-like receptor 4 contributes to phagocytosis of Salmonella Enterica serovar typhimurium via phosphoinositide 3-Kinase signaling in sheep. Cell Physiol. Biochem. 49, 662-677. doi: 10.1159/00049 3032

Wei, S., Yang, D., Yang, J., Zhang, X., Zhang, J., Fu, J., et al. (2019). Overexpression of Toll-like receptor 4 enhances LPS-induced inflammatory response and inhibits Salmonella Typhimurium growth in ovine macrophages. Eur. J. Cell Biol. 98, 36-50. doi: 10.1016/j.ejcb.2018.11.004

Yu, S. X., Du, C. T., Chen, W., Lei, Q. Q., Li, N., Qi, S., et al. (2015). Genipin inhibits NLRP3 and NLRC4 inflammasome activation via autophagy suppression. Sci. Rep. 5:17935. doi: 10.1038/srep17935
Yu, T., Guo, F., Yu, Y., Sun, T., Ma, D., Han, J., et al. (2017). Fusobacterium nucleatum promotes chemoresistance to colorectal cancer by modulating autophagy. Cell 170, 548-563.e16. doi: 10.1016/j.cell.2017.07.008

Zanoni, I., Ostuni, R., Marek, L. R., Barresi, S., Barbalat, R., Barton, G. M., et al. (2011). CD14 controls the LPS-induced endocytosis of toll-like receptor 4. Cell 147, 868-880. doi: 10.1016/j.cell.2011.09.051

Zhan, Z., Xie, X., Cao, H., Zhou, X., Zhang, X. D., Fan, H., et al. (2014). Autophagy facilitates TLR4- and TLR3-triggered migration and invasion of lung cancer cells through the promotion of TRAF6 ubiquitination. Autophagy 10, 257-268. doi: 10.4161 /auto. 27162

Zhao, Z., Zhao, J., Xue, J., Zhao, X., and Liu, P. (2016). Autophagy inhibition promotes epithelial-mesenchymal transition through ROS/HO-1 pathway in ovarian cancer cells. Am. J. Cancer Res. 6, 2162-2177.

Zhong, Z., Umemura, A., Sanchez-Lopez, E., Liang, S., Shalapour, S., Wong, J., et al. (2016). NF-kappaB restricts inflammasome activation via elimination of damaged mitochondria. Cell 164, 896-910. doi: 10.1016/j.cell.2015.12.057

Zhou, R., Yazdi, A. S., Menu, P., and Tschopp, J. (2011). A role for mitochondria in NLRP3 inflammasome activation. Nature 469, 221-225. doi: 10.1038/ nature09663

Zhu, J., Yu, W., Liu, B., Wang, Y., Shao, J., Wang, J., et al. (2017). Escin induces caspase-dependent apoptosis and autophagy through the ROS/p38 MAPK signalling pathway in human osteosarcoma cells in vitro and in vivo. Cell Death Dis. 8:e3113. doi: $10.1038 /$ cddis. 2017.488

Conflict of Interest: The authors declare that the research was conducted in the absence of any commercial or financial relationships that could be construed as a potential conflict of interest.

Copyright (C) 2020 Wang, Song, Zhang, Deng, Jiao, Qi, Lian and Yao. This is an open-access article distributed under the terms of the Creative Commons Attribution License (CC BY). The use, distribution or reproduction in other forums is permitted, provided the original author(s) and the copyright owner(s) are credited and that the original publication in this journal is cited, in accordance with accepted academic practice. No use, distribution or reproduction is permitted which does not comply with these terms. 